\title{
ВЛИЯНИЕ КРИСТАЛЛИЧЕСКИХ ФОНОНОВ НА ВТОРИЧНОЕ СВЕЧЕНИЕ ЦЕНТРА ЛЮМИНЕСЦЕНЦИИ С ЛОКАЛЬНЫМ КОЛЕБАНИЕМ
}

Imbi TEHVER. KRISTALLI FOONONITE MOJU OHE LOKAALSE VONKEGA LUMINESTSENTSITSENTRI SEKUNDAARKIIRGUSELE

Imbi TEHVER. EFFECT OF CRYSTAL PHONONS UPON SECONDARY RADIATION OF THE LUMINESCENCE CENTRE WITH A LOCAL MODE

\section{(Представил В. Хижняков)}

1. Резонансное вторичное свечение (РВС) центра люминесценции в кристалле с учетом локального и кристаллических колебаний в отдельности исследовано в $\left[{ }^{1,2}\right]$. На эксперименте в случае центров с локальными колебаниями, как правило, наблюдается также их взаимодействие с кристаллическими фононами. В связи с этим представляет интерес провести одновременный учет взаимодействия центра с отмеченными колебаниями. В этом случае каждая бесфононная линия $\mathrm{PBC}$ - либо резонансного комбинационного рассеяния (КР), либо горячей люминесценции (ГЛ), либо обычной люминесценции (ОЛ) - имеет фононное крыло. Нас интересует зависимость этого спектра от частоты возбуждения: спектр РВС будет найден при возбуждении 1) в резонансе с одной из бесфононных линий и 2) в области одного фононного крыла спектра поглощения.

Для локального колебания используем одноосцилляторную модель $\left[{ }^{1}\right]$, в которой спектр РВС определяется следующим коррелятором:

$$
\begin{gathered}
A_{\lambda}\left(\mu \tau \tau^{\prime}\right)=\mathrm{e}^{-2 \xi^{2}} \exp \left\{\xi ^ { 2 } \left[\mathrm{e}^{i \omega_{1} \mu-\Gamma|\mu|}\left(\mathrm{e}^{i \omega_{2} \tau^{\prime}-\Gamma \tau^{\prime}}-1\right) \times\right.\right. \\
X\left(\mathrm{e}^{-i \omega_{2} \tau-\Gamma \tau}-1\right)+\mathrm{e}^{i \omega_{2} \tau^{\prime}-\Gamma \tau^{\prime}}+\mathrm{e}^{-i \omega_{2} \tau-\Gamma \tau}+\mathrm{e}^{i \omega_{2}\left(\mu+\tau^{\prime}-\tau\right)} \times \\
\left.\left.\times\left(\mathrm{e}^{-\Gamma\left|\mu+\tau^{\prime}-\tau\right|}+\mathrm{e}^{-\Gamma\left(|\mu|+\tau^{\prime}+\tau\right)}-\mathrm{e}^{-\Gamma\left(\left|\mu+\tau^{\prime}\right|+\tau\right)}-\mathrm{e}^{-\Gamma\left(|\mu-\tau|+\tau^{\prime}\right)}\right)\right]\right\} .
\end{gathered}
$$

Этот коррелятор учитывает изменение положения равновесия $\xi$ и частоты $\Delta \omega=\omega_{2}-\omega_{1}$ локального колебания при электронном переходе, а также ангармоническое затухание его со скоростью Г. При этом считаем, что $\Delta \omega \gg \Gamma$.

Влияние кристаллических колебаний можно рассмотреть, если умножить $A_{\lambda}\left(\mu \tau \tau^{\prime}\right)$ на коррелятор $\left[{ }^{2}\right]$

$$
\begin{gathered}
A_{\varkappa}\left(\mu \tau \tau^{\prime}\right)=\exp \left\{g_{\varkappa}\left(\tau^{\prime}\right)+g_{\varkappa}(-\tau)+g_{\varkappa}\left(\mu+\tau^{\prime}-\tau\right)+g_{\varkappa}(\mu)-\right. \\
\left.-g_{\varkappa}\left(\mu+\tau^{\prime}\right)-g_{\varkappa}(\mu-\tau)\right\}, \\
g_{\varkappa}(x)=\sum_{i} s_{i}^{2}\left(\mathrm{e}^{i \omega_{t} x}-1\right)=\int_{-\infty}^{\infty} d \omega S^{2}(\omega)\left(\mathrm{e}^{i \omega x}-1\right), \\
s^{2}(\omega)=\sum_{i} s_{i}^{2} \delta\left(\omega-\omega_{i}\right),
\end{gathered}
$$


учитывающий кристаллические колебания в гармоническом приближении, с изменением лишь положений равновесия $\left(s_{i}\right)$ этих колебаний при электронном переходе.

Рассматриваемый спектр РВС определяется следующим интегралом (см. $\left.\left[{ }^{2}\right]\right)$ :

$$
\begin{aligned}
W\left(\omega_{0}, \Omega\right)= & \frac{B}{2 \pi} \int_{-\infty}^{\infty} d \mu \mathrm{e}^{i\left(\Omega-\omega_{0}\right) \mu} \int_{0}^{\infty} \int_{0} d \tau d \tau^{\prime} \mathrm{e}^{i\left(\omega_{0}-\Omega_{21}\right)\left(\tau-\tau^{\prime}\right)} \times \\
& \times \mathrm{e}^{-\gamma / 2\left(\tau+\tau^{\prime}\right)} A_{\lambda}\left(\mu \tau \tau^{\prime}\right) A_{\varkappa}\left(\mu \tau \tau^{\prime}\right)
\end{aligned}
$$

где $\omega_{0}$ и $\Omega$ - частоты возбуждения и $\mathrm{PBC}, \Omega_{21}$ - частота чисто-электронного перехода, $\gamma$ - радиационная ширина возбужденного электронного состояния, $B-$ нормировочный фактор $\left[{ }^{2}\right]$.

При изучении зависимости спектра РВС от $\omega_{0}$ надо иметь в виду, что актуальная область интегрирования по $\mu+\tau^{\prime}-\tau$ определяется обратной шириной $\sigma^{-1}$ спектра поглощения, по $\mu-$ обратной шириной рассматриваемой области РВС, а переменная $t=\left(\tau+\tau^{\prime}\right) / 2$ есть время пребывания системы в возбужденном (промежуточном) состоянии, интегрирование по которой в области $t \leqslant\left|\mu+\tau^{\prime}-\tau\right| \lesssim \mu$ дает релеевское рассеяние (РP) и КР, а в области $\left|\mu+\tau^{\prime}-\tau\right|,|\mu|-$ ОЛ и ГЛ $\left[{ }^{2,3}\right]$. Мы будет считать, что ширины бесфононных линий гораздо у́же ширин их фононных крыльев. Поэтому актуальные области интегрирования по $\mu, \tau$ и $\tau^{\prime}$ существенно зависят от того, где находятся частоты возбуждения или илучения: в области бесфононной линии или в области фононного крыла.

2. Спектр РВС при возбуждении в резонансе с бесфононной линией спектра поглощения находится тривиально: интенсивность бесфононных линий КР, ГЛ и ОЛ, вычисленная с учетом лишь локального колебания (см. $\left.\left[{ }^{1}\right]\right)$, умножается на фактор $\exp \left\{-2 \sum_{i} s_{i}{ }^{2}\right\}$. Действительно, в рассматриваемых областях поглощения и РВС актуальные значения $\mu, \tau, \tau^{\wedge}$ большие $\left(\sim \Gamma^{-1}\right)$, так что можно ограничиться асимптотикой $\lim _{x \rightarrow \infty} g_{x}(x)=-\sum_{i} s_{i}{ }^{2}$ и, следовательно,

$$
A_{\times}^{00}\left(\mu \tau \tau^{\prime}\right) \simeq \exp \left\{-2 \sum_{i} s_{i}^{2}\right\}
$$

Фононные крылья отмеченных линий при таком возбуждении также легко находятся: в этом случае актуальные $\mu \sim \bar{\omega}_{\varkappa}^{-1} \ll \tau, \tau^{\prime} \sim \Gamma^{-1}\left(\bar{\omega}_{x}-\right.$ средняя частота кристаллических колебаний) и поэтому

$$
A_{\varkappa}^{0 \Phi}\left(\mu \tau \tau^{\prime}\right) \simeq \mathrm{e}^{-\sum s_{t}^{2}} \exp \left\{g_{\kappa}(\mu)\right\}
$$

В результате спектр РВС в области фононного крыла при рассмотренном возбуждении определяется сверткой

$$
W\left(\omega_{0}, \Omega\right)=\mathrm{e}^{-\Sigma s_{t}^{2}} \int_{-\infty}^{\infty} d \omega W_{\chi}(\omega) W_{\lambda}(\Omega-\omega)
$$

спектра люминесценции кристаллических колебаний

$$
W_{\chi}(\Omega)=\int_{-\infty}^{\infty} d \mu \mathrm{e}^{i \Omega \mu+g_{x}(\mu)}
$$


и спектра РВС локального колебания [ $\left.{ }^{1}\right]$

$$
\begin{gathered}
W_{\lambda}\left(\omega_{0}, \Omega\right)=\frac{B}{2 \pi} \cdot \frac{x_{M}\left(\omega_{0}\right)}{\gamma+} \frac{\left|\left\langle L_{2} \mid L_{1}\right\rangle\right|^{2}}{\Gamma L_{2}}\left\{\frac{\Gamma L_{1} \delta_{L_{2} M}}{\left(\Omega-\omega_{0}+\omega_{1} L_{1}\right)^{2}+\left(\Gamma L_{1}\right)^{2}}+\right. \\
\left.+\frac{\left(1-\delta_{L_{2} M}\right)\left[\gamma+2 \Gamma\left(L_{1}+L_{2}\right)\right]}{\left(\Omega-\Omega_{21}+\omega_{1} L_{1}-\omega_{2} L_{2}\right)^{2}+\left[\Gamma\left(L_{1}+L_{2}\right)+\gamma / 2\right]^{2}}\right\} .
\end{gathered}
$$

Здесь $x_{M}-$ вероятность поглощения при возбуждении в области $M$-го вибронного повторения чисто-электронной линии $\left(\omega_{0} \simeq \Omega_{21}+\omega_{2} M\right)$, $L_{2}$ и $L_{1}$ - номера исходного и конечного уровней радиационного перехода, $\left\langle L_{2} \mid L_{1}\right\rangle$ - фактор Франка-Кондона; первый член в фигурных скобках описывает линии РP $\left(L_{1}=0\right)$ и КР $\left(L_{1}>0\right)$, второй - линии ГЛ $\left(M>L_{2}>0\right)$ и ОЛ $\left(L_{2}=0\right)$.

3. Спектр РВС при возбуждении в фононном крыле спектра поглощения несколько сложнее. Рассмотрим прежде всего КР. В этом случае актуальные $\tau, \tau^{\prime} \sim \bar{\omega}_{\varkappa}^{-1} \ll \Gamma^{-1}$, т. е. в корреляторе $A_{\lambda}\left(\mu \tau \tau^{\prime}\right)$ можно считать $\Gamma \tau \approx \Gamma \tau^{\prime} \approx 0$ :

$A_{\lambda}\left(\mu \tau \tau^{\prime}\right) \approx \mathrm{e}^{-2 \xi^{2}} \exp \left\{\xi^{2}\left[\mathrm{e}^{i \omega_{1} \mu-\Gamma|\mu|}\left(\mathrm{e}^{i \omega_{2} \tau^{\prime}}-1\right)\left(\mathrm{e}^{-i \omega_{2} \tau}-1\right)+\mathrm{e}^{i \omega_{2} \tau^{\prime}}+\mathrm{e}^{-i \omega_{2} \tau}\right]\right\}$.

Этот коррелятор дает нам рассеяние на локальном колебании. Если же учитывать рассеяние и на кристаллических колебаниях (ограничимся здесь однофононным рассеянием), то (8) надо умножить на

$A_{\varkappa}^{(1)}\left(\mu \tau \tau^{\prime}\right)=\exp \left[g_{x}\left(\tau^{\prime}\right)+g_{x}(-\tau)\right] \int_{-\infty}^{\infty} d \omega S^{2}(\omega) \mathrm{e}^{i \omega \mu}\left(\mathrm{e}^{i \omega \tau^{\prime}}-1\right)\left(\mathrm{e}^{-i \omega \tau}-1\right)$

(см. $\left.\left[{ }^{2}\right]\right)$. В результате интенсивность линии КР p-го порядка по локальному колебанию и первого порядка по кристаллическому фонону определяется формулой

$$
\begin{gathered}
W_{\mathrm{KP}}\left(\omega_{0}, \Omega\right)=\frac{B}{2 \pi} \cdot \frac{\xi^{-2 p}}{p !} \int_{-\infty}^{\infty} d \mu \mathrm{e}^{i\left(\Omega-\omega_{0}\right) \mu} \mathrm{e}^{i \omega_{1} p \mu-\Gamma p|\mu|} \times \\
X \int_{-\infty}^{\infty} d \omega S^{2}(\omega) \mathrm{e}^{i \omega \mu}\left|\int_{0}^{\infty} d \tau \mathrm{e}^{g_{\lambda}(\tau)}\left(g_{\lambda}(\tau)\right)^{p}\left\langle S_{\varkappa}(\tau)\right\rangle\left(\mathrm{e}^{i \omega \tau}-1\right)\right|^{2},
\end{gathered}
$$

где

a

$$
g_{\lambda}(x)=\xi^{2}\left(\mathrm{e}^{i \omega_{2} x}-1\right),
$$

$$
\left\langle S_{x}(x)\right\rangle=\exp \left\{-i\left(\omega_{0}-\Omega_{21}\right) x+g_{x}(x)\right\}
$$

- Фурье-образ фононных крыльев в спектре поглощения.

Покажем, что

$$
\frac{\xi^{-p}}{\sqrt{p !}} \exp \left\{g_{\lambda}(\tau)\right\}\left[g_{\lambda}(\tau)\right]^{p}=\sum_{m=0}^{\infty}\langle 0 \mid m\rangle\langle m \mid p\rangle \mathrm{e}^{i \omega_{2} m \tau},
$$

где интегралы Франка-Кондона

$$
\begin{aligned}
& \langle 0 \mid m\rangle=\mathrm{e}^{-\xi^{2} / 2 \xi m} / \sqrt{m !}, \\
& \langle m \mid p\rangle=\mathrm{e}^{-\xi^{2} / 2 \xi m+p} \sqrt{m ! p !} \sum_{i=0}^{p}(-1)^{i} \frac{\xi^{-2 i}}{i !(p-i) !(m-i) !} .
\end{aligned}
$$

Для этого преобразуем 


$$
\exp \left[g_{\lambda}(\tau)\right]\left[g_{\lambda}(\tau)\right]^{p}=\sum_{k=0}^{\infty} \frac{\xi^{2(k+p)}}{k !} \sum_{m=0}^{k+p}(-1)^{k+p-m}\left(\begin{array}{c}
k+p \\
m
\end{array}\right) \mathrm{e}^{i \omega_{2} m \tau}
$$

Переходя к новому индексу суммирования $j=k+p-m$, имеем

$$
\begin{aligned}
& \sum_{m=0}^{\infty} \frac{\xi^{2 m}}{m !} \mathrm{e}^{i \omega_{2} m \tau} \sum_{j=0}^{\infty}(-1)^{j} \frac{\xi^{2 j}}{j !} \frac{(m+j) !}{(m-p+j) !}= \\
= & \sum_{m=p}^{\infty} \frac{\xi^{2 m}}{(m-p) !} \mathrm{e}^{i \omega_{2} m \tau_{1} F_{1}}\left(m+1, m+1-p ;-\xi^{2}\right),
\end{aligned}
$$

где ${ }_{1} F_{1}(\alpha, \gamma ; z)$ - вырожденная гипергеометрическая функция. Для доказательства формулы (11) остается учесть, что ${ }_{1} F_{1}(\alpha, \alpha ; z)=\mathrm{e}^{z^{2}}$ и

$$
(z / \gamma){ }_{1} F_{1}(\alpha+1, \gamma+1 ; z)={ }_{1} F_{1}(\alpha+1, \gamma ; z)-{ }_{1} F_{1}(\alpha, \gamma ; z),
$$

откуда

$$
\frac{\xi^{2 m}}{(m-p) !}{ }_{1} F_{1}\left(m+1, m+1-p ;-\xi^{2}\right)=\langle 0 \mid m\rangle\langle m \mid p\rangle \xi^{p} \sqrt{p !} .
$$

Проинтегрируем теперь (10). Интеграл по $\tau$

$$
\int_{0}^{\infty} d \tau\left\langle S_{x}(\tau)\right\rangle\left(\mathrm{e}^{i \omega \tau}-1\right) \mathrm{e}^{i \omega_{2} m \tau}=\Phi\left(\omega_{0}-\omega_{2} m-\omega\right)-\Phi\left(\omega_{0}-\omega_{2} m\right)
$$

выражается через комплексную функцию показателя преломления

$$
\Phi(x)=\oint_{-\infty}^{\infty} d_{\omega} \frac{x(\omega)}{\omega-x}+i \pi x(x)
$$

где $x(x)$ - форма фононных крыльев в спектре поглощения.

Проинтегрировав и по $\mu$, получим, что при возбуждении в фононном крыле $m$-го вибронного повторения чисто-электронной линии интенсивность линии КР p-го порядка по локальному колебанию и первого порядка по кристаллическому фонону определяется формулой

$$
\begin{aligned}
& W_{\mathrm{KP}}\left(\omega_{0}, \Omega\right)=\frac{B}{2 \pi} \int_{-\infty}^{\infty} d_{\omega} s^{2}(\omega) \frac{2 \Gamma p}{\left(\Omega-\omega_{0}+\omega_{1} p+\omega\right)^{2}+(\Gamma p)^{2}} \times \\
& X\left|\sum_{m=0}^{\infty}\langle 0 \mid m\rangle\langle m \mid p\rangle\left[\Phi\left(\omega_{0}-\omega_{2} m-\omega\right)-\Phi\left(\omega_{0}-\omega_{2} m\right)\right]\right|^{2} .
\end{aligned}
$$

В этой формуле сумма по $m$ учитывает интерференцию разных каналов рассеяния с участием различного числа виртуальных квантов локального колебания. В рассматриваемом случае возбуждения в фононном крыле ее учет существен, поскольку ширина фононного крыла, как правило, сравнима с частотой локального колебания $\omega_{2}$. Если же ширина фононных крыльев гораздо у́же $\omega_{2}$, то достаточно учесть вклад лишь резонансного вибронного перехода в комплексный показатель преломления, положив $m=M$. Тогда формула (15) существенно упрощается:

$$
\begin{aligned}
& W_{\mathrm{KP}}\left(\omega_{0}, \Omega\right)=\frac{B}{2 \pi} \int_{-\infty}^{\infty} d \omega s^{2}(\omega) \frac{2 \Gamma p}{\left(\Omega-\omega_{0}+\omega_{1} p+\omega\right)^{2}+(\Gamma p)^{2}} \times \\
& \times|\langle 0 \mid M\rangle\langle M \mid p\rangle|^{2}\left|\Phi\left(\omega_{0}-\omega_{2} M-\omega\right)-\Phi\left(\omega_{0}-\omega_{2} M\right)\right|^{2} .
\end{aligned}
$$


Рассмотрим в заключение линии ОЛ и ГЛ, возбуждаемые в фононном крыле. В этом случае актуальные значения $t \geqslant \Gamma^{-1} \gg \bar{\omega}_{\varkappa}^{-1}$. Следовательно, коррелятор $A_{\varkappa}\left(\mu \tau \tau^{\prime}\right)$ можно заменить его асимптотическим значением [ $\left.{ }^{2}\right]$

$$
\begin{gathered}
\lim _{t \rightarrow \infty} A_{x}\left(\mu \tau \tau^{\prime}\right)=\exp \left[g_{x}(\mu)+g_{x}\left(\mu+\tau^{\prime}-\tau\right)\right]= \\
=\int_{-\infty}^{\infty} d \omega F(\omega) \mathrm{e}^{i \omega \mu} \int_{-\infty}^{\infty} d \omega^{\prime} F\left(\omega^{\prime}\right) \mathrm{e}^{i \omega^{\prime}\left(\mu+\tau^{\prime}-\tau\right),}
\end{gathered}
$$

где

$$
F(\omega)=s^{2}(\omega)+\frac{1}{2} \int_{-\infty}^{\infty} d x s^{2}(x) s^{2}(\omega-x)+\ldots .
$$

Подставляя (17) в (4) и учитывая (1), получаем, что рассматриваемый спектр выражается в виде сверток

$$
W_{\text {ол }+г л}\left(\omega_{0}, \Omega\right)=\frac{B}{2 \pi} \int_{-\infty}^{\infty} d \omega^{\prime} F\left(\omega^{\prime}\right) \varkappa_{M}\left(\omega_{0}-\omega^{\prime}\right) \times
$$

Ees?

$$
\times \int_{-\infty}^{\infty} d_{\omega} F(\omega) \frac{\left(1-\delta_{L_{2} M}\right)\left[\gamma+2 \Gamma\left(L_{1}+L_{2}\right)\right]\left|\left\langle L_{2} \mid L_{1}\right\rangle\right|^{2}}{\left(\Omega-\Omega_{21}+\omega_{1} L_{1}-\omega_{2} L_{2}+\omega\right)^{2}+\left[\Gamma\left(\vec{L}_{1}+L_{2}\right)+\gamma / 2\right]^{2}} .
$$

Автор признателен Л. Ребане и В. Хижнякову за обсуждение данной проблемы.

\section{ЛИ Т Е РА Т У Р А}

1. Hizhnyakov, V., Tehver, I., Phys. status solidi (b), 82, № 1, K89-K93 (1977).

2. Hizhnyakov, V., Tehver, I., Phys. status solidi, 21, № 2, 755-768 (1967).

3. Reba ne, K. K., Tehver, I. Y., Hizhnyakov, V. V., In: The Theory of Light Scattering in Solids, ed. by V. Agranovich and J. Birman, M., «Nauka», 1976, p. $467-486$.

\section{Институт физики \\ Академии наук Эстонской ССР}

